\title{
Multiple Realization in Systems Biology
}

\begin{abstract}
Polger and Shapiro (2016) claim that unlike human-made artifacts cases of multiple realization in naturally occurring systems are uncommon. Drawing on cases from systems biology, I argue that multiple realization in naturally occurring systems is not as uncommon as Polger and Shapiro initially thought. The relevant cases, which I draw from systems biology, involve generalizable design principles called network motifs which recur in different organisms and species and perform specific functions. By examining two network motifs, negative autoregulation and feed-forward loops in detail, I show that network motifs with entirely different underlying causal structures can perform the same function of interest. The article also considers the scope problem of multiple realization, namely, are cases of MR in the biological world rare?
\end{abstract}




\section{Introduction}

Multiple realization (MR) is the thesis that, roughly speaking, a higher-level scientific kind can be multiply realized (or is at least multiple realizable) by more basic scientific kinds (Shapiro 2000). ${ }^{1}$ The thesis was initially proposed to describe the relationship between psychological and neuroscientific kinds (Putnam 1967; Block and Fodor 1972; Fodor 1974), but was later extended to fields such as philosophy of biology (Kitcher 1984; Sober 1999; Koskinen 2017; Fang 2018). For many participants in the debate (e.g., Polger and Shapiro 2016), MR is essentially an empirical thesis concerning a particular type of phenomenon happening in the real world and, as such, claims for (or against) it must be supported by empirical evidence. Philosophical interest in this thesis largely stems from its close relation to many important philosophical questions, such as the autonomy of the special sciences (e.g., Fodor 1974; Rosenberg 2001), reductionism (e.g., Sober 1999; Bickle 2003), functions and functional properties (e.g., Couch 2005; Chirimuuta 2018),

1 The terms multiple realization and multiple realizability are sometimes used loosely or interchangeably. As Polger and Shapiro (2018) clarify, the former refers to the fact that some property, kind or function is actually multiply realized while the latter refers to the possibility that some property, kind or function can be multiply realized. As this paper evaluates the extent to which kinds are actually realized, I will be using the term multiple realization (or its abbreviation, MR). 
etc.

Many attempts have been made to refine, defend and refute the MR thesis (Bechtel and Mundale 1999; Clapp 2001; Polger 2002, 2008, 2015; Bickle 2003, 2010; Gillett 2003; Couch 2004, 2009; Richardson 2008; Sullivan 2008). However, perhaps the most intriguing development in the last two decades owes to Lawrence Shapiro's body of work (2000, 2004, 2008; see also Polger and Shapiro 2016). A central component of this work is Shapiro's causally relevant differences criterion (2000). According to this criterion, two cases count as instances of MR only when they contribute causally relevant differences to the realization of the same (or similar) property or function. ${ }^{2}$ This criterion captures the heart of the MR thesis and arguably provides a framework in which vague intuitions, grounded solely in conceptual possibilities, can give way to detailed analysis of concrete scientific evidence.

Nonetheless, Shapiro's criterion was somewhat underdeveloped. Based on recent advances in the literature (and especially on what Shapiro and Polger have accomplished during the last two decades), a well-articulated official recipe for judging cases of MR has been put forward (Polger and Shapiro 2016). This recipe has a number of merits, not only

\footnotetext{
${ }^{2}$ Following Polger and Shapiro (2016), I will take a neutral stance towards the relata of the realization relationship, should it be properties, kinds, or functions. I will say more about kinds and functions in the following sections.
} 
because it incorporates the key insight contained in the causally relevant differences criterion, but also respects a couple of widely agreed upon aspects of realization. For example, the recipe admits that MR involves different taxonomic systems for determining the sameness and/or difference of properties or functions; that different taxonomic systems may have distinct criteria of sameness and/or difference with respect to properties/functions $(2016,62) .^{3}$

With this full-fledged recipe in place, many supposed cases of MR, such as pain in humans and octopuses, neuroplasticity, compound vs. camera eyes, etc., can be easily evaluated and adjudicated. However, there is a curious discontinuity here, as Polger and Shapiro arrive at different conclusions when examining cases from artifacts and naturally occurring systems. While granting the existence of MR in naturally occurring systems, Shapiro and Polger maintain that MR is much more common in artifacts:

3 This point constitutes a challenge to Bechtel and Mundale's (1999) objection to the MR thesis, because they require in their argument that the MR thesis really holds only when the realizer and the realized employ the same grain size in their taxonomies. However, first, it is a matter of fact that different sciences usually do not employ the same grain size in their taxonomies, and second, as Zerrilli (2017) recently has pointed out, Bechtel and Mundale's request for the sameness of grain size across different taxonomic systems is impossible to follow at best and misleading at worst. 
We turn to these examples [artifacts] because multiple realization is much less common in naturally occurring systems than is usually recognized. The conditions for the kinds of variation that count as multiple realization are easy to create at a workbench or drafting table, but they are relatively rare in nature. $(2016,74)$

They arrive at this conclusion by denying several supposed cases of MR, such as neuroplasticity, memory, and the conceptual possibility of artificial intelligence.

The purpose of this essay is to show that MR occurs in the biological world, and more often than Polger and Shapiro, along with many other philosophers, envision. ${ }^{4}$ I defend this claim using a detailed analysis of concrete examples drawn from systems biology. Systems biology is a thriving new discipline that enjoys both an engineering and biological perspective, in which general design principles (called 'network motifs') that recur in

${ }^{4}$ Koskinen (2017) and Chirimuuta (2018) recently have also argued for the common existence of MR in biological systems. Koskinen approaches this issue from the perspective of synthetic biology, where scientists not only create novel functional realizations that are not true of the actual biological world, but also streamline functional realizations that could otherwise come to be true in the actual biological world. Chirimuuta highlights the centrality and prevalence of functional thinking in biology, arguing for a biologically-grounded conception of multiple realization that does a better job in recognizing the importance of functional thinking in biology. 
different organisms and species are being discovered. The interdisciplinary nature of this new area shows that the gap between artifacts and naturally occurring systems is in fact not as large as many would imagine.

The essay unfolds as follows. Section 2 sets the stage by unpacking Polger and Shapiro's official recipe. Section 3 describes two generalizable design principles (i.e., network motifs) in systems biology that serve the same function of boosting the response time of transcription networks. Section 4 examines the question of whether the two network motifs constitute a genuine case of MR in terms of the official recipe. Finally, Section 5 argues that MR occurs far more commonly in the biological world than Polger and Shapiro give it credit for.

\section{Polger and Shapiro's Official Recipe}

To determine when there is a case of MR, Shapiro (2000) suggests a useful causally relevant differences (CRD) criterion. According to the CRD criterion:

Multiple realizations count truly as multiple realizations when they differ in causally relevant properties - in properties that make a difference to how they contribute to the capacity under investigation. (Shapiro 2000, 644; author's emphasis) 
The key to this criterion is that, as suggested by Shapiro's slogan "same but different" (Shapiro 2000, 2004, 2008), different realizers must contribute causally relevant differences to the realization of the same (or at least similar) property or function. That is, multiple realization is a situation wherein causally relevant differences result in sameness in the realized property or function. By contrast, if the sameness of the realized property or function can be causally traced back to the sameness of the realizers, and if the differences can also be causally traced back to the differences of the realizers, then the realizers are not genuinely different realizers. Only those cases where differences result in sameness can count as cases of multiple realization. A well-known example helps illustrate this point. Two waiter's corkscrews can do the job of removing corks equally well, even though one is red and the other is blue. Do these two corkscrews count as different realizers of the same function of removing corks? Certainly not, because the difference in color does not contribute to how they realize the function of removing corks. How about a slightly different scenario where one waiter's corkscrew is made of aluminum and another is made of steel? The answer is still negative, for again the difference in material composition in this case does not seem to contribute to how they realize the function of interest.

One might wonder, then, if there is any way for two corkscrews to be different realizers of the same function of removing corks. This time the answer is affirmative. One 
way discussed by Shapiro (2000) is associated with different mechanical principles whereby different types of corkscrews remove corks in different ways. For example, a waiter's corkscrew differs from a winged corkscrew in that they utilize different mechanical principles to remove corks, even though they might be of the same color or made of the same material.

However, there remain puzzling cases that the CRD criterion does not seem to accommodate so easily. On the face of it, we might think that a difference in material composition is not causally relevant to removing corks. But that is not exactly right. For instance, a difference in material composition can lead to a difference in rigidity, which in turn can lead to a difference between failing and succeeding in removing corks. Corkscrews made of plastic, for example, might be too soft to remove any corks. So, it seems that the difference in rigidity due to the different material composition is causally relevant after all. Therefore, according to the CRD criterion, the realizers that contribute causally relevant differences to the realization of a function do in fact constitute different realizers.

To disentangle puzzles of this sort, the CRD criterion needs clarification. First, it is important to the criterion that the same property or function is realized in different ways (Polger and Shapiro 2016, 67). In terms of this condition, a waiter's corkscrew that is soft will work in the same way as one that is rigid, albeit less efficiently. The waiter's 
corkscrews do not, therefore, count as cases of MR. Further, the different efficiency in removing corks due to their difference in rigidity is an individual variation rather than a difference in kind. Moreover, we must be clear that MR is relational, as opposed to absolute and transitive, such that we should specify what exactly is the realizer and what exactly is the realized when evaluating putative cases of MR (Polger 2008). For instance, with respect to the property of rigidity, corkscrews with different material composition may count as cases of MR because they sometimes result in the same rigidity in different ways. ${ }^{5}$ In contrast, with respect to removing corks, different material composition cannot result in MR since the compositional variations on a given corkscrew design are causally irrelevant to the mechanism of cork removal.

Taking account of these considerations, and integrating the body of work on MR they have developed over the last two decades (Shapiro 2004, 2008; Polger and Shapiro 2008; Polger 2009; Shapiro and Polger 2012), Polger and Shapiro have proposed an updated official recipe for MR (Polger and Shapiro 2016). According to this recipe, two instances (or entities, objects, properties, etc.) constitute a case of MR only if they meet the following conditions:

\footnotetext{
5 E.g., metals and woods are rigid in different ways, one involving isotropic molecular arrangement while another involving anisotropic laminar cellular arrangement.
} 
(a) They are of the same kind in a higher taxonomic system or model;

(b) They are of different kinds in a lower taxonomic system or model;

(c) The factors that lead the two instances to be differently classified by the lower taxonomic system must be among those that lead them to be commonly classified by the higher taxonomic system;

(d) The relevant variation between the two instances, according to the lower taxonomic system, must be distinct from the intra-kind variation between the two instances according to the higher taxonomic system (adapted from Polger and Shapiro 2016, 68).

The two instances are bearers of the functions or functional kinds defined by the special sciences such as systems biology, wherein a function typically refers to what something — be it an entity, a state, a property, or an object — can do (Polger and Shapiro 2016, 22). ${ }^{6}$ Alternatively, the two instances are instantiations of two causal mechanisms or principles. Notice that MR involves different taxonomic systems, defined by different sciences, explanations, or models (Ibid., 26-32; see also Couch 2004; Polger 2008; Richardson 2008). Consequently, the sameness or difference of functions is judged by their

${ }^{6}$ Fodor (1974) thinks that special sciences kinds are usually functionally individuated kinds, with which Shapiro agrees (2000, 2004, 2016). 
own taxonomic systems in their own sciences, explanations, or models, respectively. In other words, “[...] sameness and difference are understood relative to taxonomies" (Polger and Shapiro 2018, 449). Condition (c) says that two instances should be different in the way that they realize the same function in different ways, where the different ways can be classified by the lower taxonomic system and the same function by the higher one. Finally, condition (d) attempts to make the distinction between intra-kind variations and inter-kind differences, where inter-kind differences are determined by whether they accomplish a function in different ways using the lower taxonomic system. Note that this recipe differs from the CRD criterion in one important aspect: it does not require that the factors that lead the two instances to be differently or commonly classified (by the lower or higher taxonomic system, respectively) be causal. The factors can be causal or non-causal. ${ }^{7}$ However, for this essay's purpose I need only focus on the causal situation.

With this updated official recipe at hand, we are in a good position to evaluate supposed cases of MR. For example, it is not so hard to show that the case of waiter's corkscrews with different rigidity cannot constitute a case of MR, for it simply does not

7 This renders the recipe much broader to perhaps include non-causal forms of MR, e.g., compositional MR suggested by Aizawa and Gillett (2011): compositional differences generate MR in the way that the compositional differences that matter for the sake of the realizer-level classification are also among those responsible for the realized-level similarity. 
satisfy condition (c); namely, it is not the case that the difference in rigidity leads to different modes of realization. By contrast, a waiter's corkscrew and a winged corkscrew do count as different ways of realizing the same function. First, suppose there is a special science of corkscrews. Such an imagined science might say that the waiter's and winged corkscrews represent two distinct kinds since they employ different mechanical principles to do the same thing, i.e., removing corks. So, the second condition is satisfied. Moreover, such a science might also say that, in terms of the job these two kinds of corkscrews perform, they are better classified as being of the same kind. Note that this imaginary science has deployed two taxonomic systems when judging whether the waiter's and winged corkscrews belong to the same kind: one relating to mechanical principles, and the other to the common goal they achieve. Hence, the first condition is also met. Furthermore, the different mechanical principles embody causally relevant differences that lead the two kinds of corkscrews to fulfil the same goal; in other words, to borrow Shapiro's slogan, it is the differences that result in the sameness. The the third condition is thus met. Finally, as implied by the fulfilment of the second condition, the differences between the waiter's and winged corkscrews are clearly inter-kind differences, and any differences in how efficiently these artifacts achieve the same goal constitute an intra-kind variation. Therefore, the last condition is also satisfied. Taken together, we can safely conclude that this is a case of MR. 


\section{Design Principles in the Biological World ${ }^{8}$}

Artifacts such as corkscrews and watches are largely uncontroversial. The consensus partly arises from the observation that human designers usually intentionally build artifacts that comply with different design principles and yet serve the same purpose. There is less agreement over whether cases of MR are also common in naturally occurring systems such as biological systems, where no intentional designers can be found. Some hold that MR is common in naturally occurring systems (Gillett 2003; Sullivan 2008; Aizawa 2009, 2013; Fang 2018), whereas others refute (Shapiro 2000, 2004, 2008; Shapiro and Polger 2012; Polger and Shapiro 2016).

However, recent achievements and developments in systems biology have shown that the gap between artifacts and biological systems might be not as sharp as many have imagined. Despite the astonishing complexity of the biological world, it is possible to find

${ }^{8}$ Note that design principles have recently drawn a lot of attention from philosophers concerned with systems biology (e.g., Braillard 2010; Levy and Bechtel 2013; Green 2015, 2017; Brigandt 2018). Even though these authors do not explicitly discuss MR, they all highlight that design principles are abstracted away from systems with heterogeneous underlying causal details. Namely, their discussions on design principles seem to support MR. 
surprisingly simple design principles that can be applied to a wide variety of biological systems, over a whole range of different species. ${ }^{9}$ More remarkably, some design principles with entirely different underlying causal mechanisms can sometimes accomplish the same goals, e.g., boosting the response time of transcription networks in response to environmental signals.

Before proceeding, a few words on systems biology are in order. One of the central goals of this subdiscipline is to search for generalizable patterns or principles that arise from interactions between various constituents (e.g., molecules) of living organisms. Importantly, the patterns they search for are those that cannot be discovered by focusing on the constituents of living organisms alone (Alberghina and Westerhoff 2005; Alon 2007a). The advent of large-scale modeling and experimentation techniques (e.g., computational simulation) as well as the ready availability of high throughput data ranging over multiple scales/levels, make the searching for these generalizable principles an achievable goal (Kitano 2002; O’Malley and Dupré 2005; Boogerd et al. 2007).

Thanks to the efforts taken during the last two decades, systems biologists have started to realize that, within the extremely complicated networks of interactions observed

\footnotetext{
${ }^{9}$ Design principles here do not imply that there are human designers. They are just results of natural selection whereby advantageous features were selected and preserved while disadvantageous features were simply washed out.
} 
in organisms, there seem to be simple building-block patterns that recur in different organisms and species. These building-block patterns, called network motifs, refer to "Patterns that occur in the real network significantly more often than in randomized networks" (Milo et al., 2002; Shen-Orr et al., 2002; Cf. Alon 2007a, 27). ${ }^{10}$ The reason that network motifs appear more often than random networks is due to the selection advantages they confer upon their bearers - that is, they must be constantly selected during evolution, a point we will revisit later.

Since one goal of systems biology is to search for generalizable design principles that underlie biological systems, it provides ground on which to assess whether the fact of attaining the same goals or fulfilling the same functions by different design principles is a sound basis for challenging Polger and Shapiro's position that MR occurs rarely in the biological world. The answer to this question, I think, is affirmative. However, to pave the way for answering this question, let me first describe two design principles discovered by systems biologists.

\subsection{Negative autoregulation}

${ }^{10}$ For example, statistical analysis shows that, for the type of network motif negative autoregulation, the difference in occurrence frequency between it and random networks is marked by 32 standard deviations, showing a high statistical significance (Alon 2007a, $30-31)$. 
First consider the type of network motif termed negative autoregulation (NAR) in sensory transcription networks, in comparison with simple regulation:
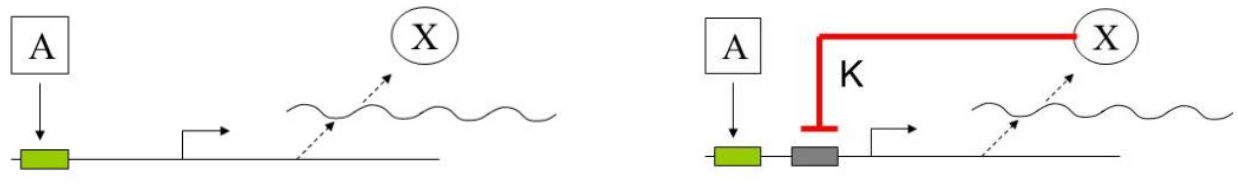

Figure 1. A comparison of simple regulation and negative autoregulation. The left is a simple regulation network while the right a negative autoregulation network. Arrows in the figure denote causal interactions such as binding, activation, while $\dashv$ represents repression. This figure comes from Alon (2007a, 30).

The left is a simple regulation network, where a transcription factor $A$ binds the promotor of gene $X$, which activates the expression of gene $X$. The transcription factor is a special kind of protein that "bind[s] to particular DNA sequences in gene regulatory regions and control[s] their transcription" (Latchman 1993, 417). The right side is a NAR network, where the gene product of $X$ binds a specific site in the promotor of gene $X$, which represses its own transcription. $K$ denotes the activation coefficient, defined as "the concentration of $X$ needed to repress the promotor activity by 50\%" (Alon 2007a, 30).

The only difference between simple regulation and NAR is an additional link, $\dashv$, 
representing the repression of a gene by its own gene products (e.g., proteins). When there is such a subtle difference, an interesting scientific question arises: why did nature select and preserve this structure rather than simply utilize the simpler regulation also available? The answer is that it provides its bearers with some important functions that the simple regulation network fails to provide. In fact, studies using mathematically controlled comparison have shown that one of the functions it confers on its bearers is boosting the response time of the transcriptional network - that is, giving a very rapid initial production rate (Savageau 1976; see also Molina et al. 1993; Rosenfeld et al. 2002). The response time is defined as "the time to reach halfway between the initial and final levels in a dynamic process" (Alon 2007a, 19), which is often expressed as $T_{1 / 2}$. The following figure shows the difference in response time between simple regulation and NAR:

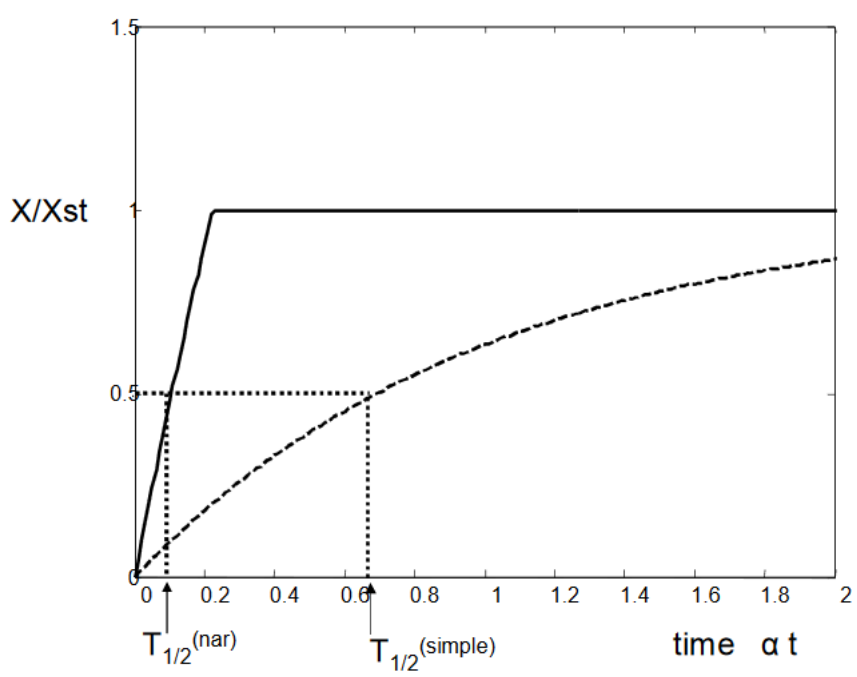


Figure 2. A comparison of response time between simple regulation (dashed line) and NAR under the condition that they arrive at the same steady-state level and have the same degradation/dilution rates, $\alpha . T_{1 / 2}^{(\text {nar })}$ denotes the response time of NAR, $T_{1 / 2}^{(\text {simple })}$ the response time of simple regulation, $X / X_{s t}$ the ratio of the level of gene product $X$ and the level of $X$ at its steady state. This figure comes from Alon (2007a, 35).

It can be seen from Figure 2 that the response time of NAR is significantly shorter than simple regulation. It also can be seen from Figure 2 that, due to the high concentration of the gene product $X$ rapidly produced within a short time interval and the repression nature of the network, the NAR network stops production shortly and quickly reaches its sought-after steady state. By contrast, because of the lack of the pulse-like fast initial production, the simple regulation network can only gradually and slowly climb onto the same steady state. Note that a simple regulation network can also reach a rapid initial production rate when equipped with a very strong promoter (or when it's gene products have a high degradation rate), but the cost would be huge since this may incur overexpression of the gene product (Rosenfeld et al. 2002, 785; Alon 2007a, 34).

Insofar as the NAR network is widespread in the biological world, there is a reason why it was selected and preserved. One possible explanation is that it conferred great 
advantages on its bearers, because rapid response time means high efficiency in producing gene products and quick response to environmental variations. ${ }^{11}$ All these obvious advantages, as shown by the comparison above, cannot be achieved by the simple regulation network.

Therefore, due to these merits that the NAR network confers upon its bearers, it is not surprising that it was selected and preserved over evolutionary time.

\subsection{The incoherent type-1 feed-forward loops}

The previous section has shown that the NAR network motif serves the function of boosting the response time of transcription networks in response to environmental signals. If boosting response time turns out to be a good strategy with which organisms can cope with environmental variations, then one can reasonably hypothesize that the same strategy might be rediscovered by other biological mechanisms over evolutionary time. As a matter of fact, the same strategy is also employed by a totally different kind of network motif with a distinct causal underpinning: the incoherent type-1 feed-forward loop (I1-FFL hereafter), as shown in Figure 3 below (Mangan and Alon 2003; Mangan et al. 2006; Alon 2007a, b).

11 The NAR network has other advantages compared with the simple regulation, for example, it can increase robustness to fluctuations in production rate (Alon 2007a, 34-37). 
For illustrative purposes, a coherent type-1 FFL is also shown below.

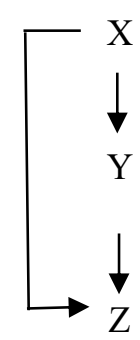

(a)

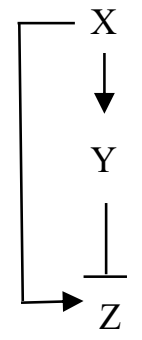

(b)

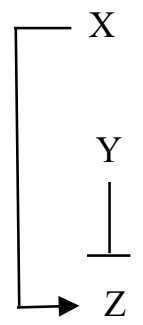

(c)

Figure 3. (a) is the coherent FFL, (b) the incoherent FFL and (c) simple regulation. Arrows represent activation while $\dashv$ represents repression. (a) is coherent because the sign of the direct path from $X$ to $\mathrm{Z}$ has the same overall sign as the indirect path from $X$ through $Y$ to $Z$. "The overall sign of a path is given by the multiplication of the sign of each arrow on the path (so that two minus signs give an overall plus sign)" (Alon 2007a, 47). Hence (b) is incoherent. This figure is adapted from Alon (2007a, 47).

Like the NAR motif, the FFL also constitutes a very common type of network in biological systems (e.g., in E. coli, yeasts, and many higher organisms including humans) (Milo et al. 
2002; Mangan and Alon 2003). ${ }^{12}$ The I1-FFL network has two transcription factors $X$ and $Y$, wherein the first transcription factor $X$ activates the second $Y$. Both $X$ and $Y$ bind the regulatory region of the target gene $Z$ and collectively regulate $Z$ 's activity. However, $X$ works as an activator while $Y$ works as a repressor for $Z$. Hence, the two paths have opposite effects on $Z$ : the direct path activates $Z$ whereas the indirect represses $Z$.

The dynamics of this network are as follows: At the beginning, when there is some input signal present, the transcription factor $X$ is activated. The activated $X$ then binds the promotor of gene $Z$, which leads to the transcription of $X$ and the production of some gene products. At the same time, $X$ also activates $Y$ when there is some input signal for $Y$. After a delay, while the concentration of $Y$ accumulates and reaches its threshold, it starts to repress the production of $Z$ 's products, hence decreasing the activity level of $Z$. All in all, the network brings about a pulse-like shape expression rate of $Z$, as shown below:

12 Research shows that the FFL's occurrence frequency is higher than random networks with the same number of nodes and edges by more than 30 standard deviations, indicating a very high statistical significance. 


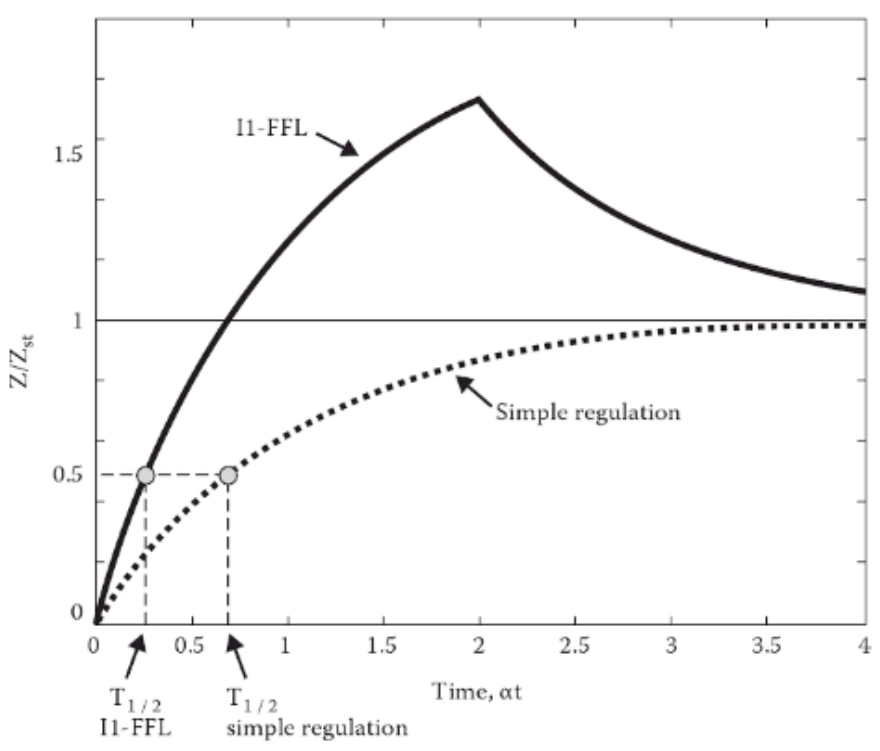

Figure 4. A pulse-like shape expression rate of $Z$. Also shown is the comparison of the response time of the I1-FFL with simple regulation. This figure comes from Alon (2007a, 61).

Figure 4 shows that the I1-FFL has a significantly shorter response time compared with simple regulation. The response time of the I1-FFL is associated with the repression factor F, representing the strength of the repression effect of $Y$ on $Z$ (Alon 2007a, 62). Hence, the greater the repression factor $\mathrm{F}$, the stronger the repression effect of $Y$ on $Z$. F is also inversely proportional to the response time - that is, the greater the repression factor F, the shorter the response time. ${ }^{13}$ Therefore, "the stronger the effect of $Y$ in repressing production of $Z$, the faster the performance of the I1-FFL compared to an equivalent

13 For detailed calculations of the response time, see Alon (2007a, 61-62). 
simple-regulation circuit $X \rightarrow Y$ made to reach the same steady-state level of $Z$ " (Ibid., $62)$.

Also, an evolutionary story can be told about why the I1-FFL was selected and preserved over evolutionary time. The explanation here is identical to that given for negative autoregulation: the I1-FFL conferred great selection advantages on its bearers because a quick initial production rate means high production efficiency and rapid response to environmental variations. ${ }^{14}$

\section{Are These Network Motifs Cases of MR?}

Having set the stage, it is time to answer the key question of this essay: whether the different design principles fulfilling the same function offer good reasons to challenge Polger and Shapiro's position that MR occurs rarely in the biological world. This question will be answered in two steps. This section will address the question of whether the network motifs described in the last section constitute a case of MR, and Section 5 will answer the question of whether MR occurs as rarely as Polger and Shapiro thought.

At a first approximation, the NAR and I1-FFL networks constitute a case of MR.

14 In addition to boosting the response time, the I1-FFL can also serve as a pulse generator (Alon 2007a, 57-62). 
They are two different designs with entirely different causal underpinnings, but which realize the same function of boosting the response time of transcription networks. Moreover, the differences between the two designs are causally relevant because it is these different causal underpinnings that contribute to the realization of the same function.

We must not, however, reach our conclusion too hastily. Instead, let us examine this case more carefully using Polger and Shapiro's official recipe. To begin with, we should be able to honor the notion of definite taxonomic systems in systems biology. The job of a taxonomic system in this context would be to classify the various networks found in living organisms into different kinds, where these kinds exhibit distinctive causal structures that recur sufficiently frequently and are associated with distinct system dynamics. Moreover, in order to be classified as kinds, they must show some degree of modularity, meaning that they can work appreciably well under conditions in which they are detached from their initial interaction networks in the cell they are embedded in as components. The condition of modularity ensures their (at least partial) independence, separability, and identity as functional units.

It turns out that the NAR and I1-FFL networks (and many other kinds of networks such as the single-input network, the multi-output feed-forward loop, the dense overlapping regulon, etc.) satisfy all these conditions for being different kinds, since each has its own distinctive causal structure (recall the causal graphs depicted in Section 3), 
recurs sufficiently commonly compared with random networks, and shows some degree of modularity. Hence, they can be classified as different kinds in this taxonomic system. In practice, systems biologists do understand the two network motifs - or design principles, more broadly — as different kinds because their distinct underlying causal mechanisms/structures are associated with heterogeneous system dynamics (see, e.g., Kitano 2002; Kollmann et al. 2005; Alon 2007a; Novák and Tyson 2008). Thus, the second condition of Polger and Shapiro's recipe is satisfied.

It remains to be seen, however, whether there is another taxonomic system in systems biology that classifies the NAR and I1-FFL networks as members of the same kind. Unfortunately, it seems that systems biology does not explicitly provide such a classificatory tool. This is understandable: systems biology is not concerned with whether there are cases of MR, and thus is not concerned, in turn, with whether we can find different taxonomic systems for differentiating realized properties and functions from their realizers. However, this does not mean that we philosophers cannot detect (or at least infer) from the practice of systems biologists that there is such a taxonomic system. It seems that systems biologists have in mind a level of description associated with the functions that organisms fulfil; let us call it the functional level. At the functional level, the primary concern is with what a network motif can actually do and, furthermore, whether the job accomplished by a motif can also be done by other motifs. Interestingly, systems biologists 
such as Uri Alon are clearly guided by these sorts of questions in their research. Alon deliberately groups three different types of motifs together in terms of their common function of boosting the response time of transcription networks; ${ }^{15}$ as he says that "We have by now seen three different ways to speed the response time of transcription networks" (Alon 2007a, 64). Therefore, we can say that the NAR and I1-FFL networks are of the same kind in a different taxonomic system, a taxonomic system defined by the function different motifs can accomplish. This meets the first condition of Polger and Shapiro's recipe. ${ }^{16}$

15 A third way to achieve this is via simple regulation not discussed in this essay. For relevant discussion see Alon (2007a, 18-22).

16 One might be cautious about reading too much into this one source. However, Alon's book (2007a) is not only his own work on the issue but also summarizes the work conducted by a number of scientists from his group, e.g., Milo et al. (2002), Rosenfeld et al. (2002), Shen-Orr et al. (2002), Mangan et al. (2003), Mangan et al. (2006), etc.

Furthermore, there are many other research groups, in addition to Alon's group, that are also engaged in exploring and identifying the various common functions linked with specific motifs. For example, Camas et al. (2006) and Zabet (2011) are concerned with negative autoregulation's function of boosting the response time of transcriptional networks, while Wall et al. (2005) and Kim et al. (2008) are concerned with the I1-FFL's function of boosting the response time. Singh and Hespanha (2009) show that negative autoregulation can serve the function of noise suppression, while Ghosh et al. (2005) show 
Now consider whether the network motifs satisfy the third condition. Recall that the third condition requires that the factors that lead the two instances (e.g., NAR and I1-FFLs) to be differently classified by a lower taxonomic system must be among those that lead them to be commonly classified by a higher taxonomic system. Let us first specify the very factors that lead NAR and I1-FFLs to be differently classified. As I said above, these factors are related to the two distinct causal underpinnings exemplified by the two network motifs. To be sure, these are totally different causal structures, representing two separate causal scenarios. The situation here is analogous to the corkscrew case, wherein the waiter's and winged corkscrews represent two separate mechanical principles - they

that the type-1 coherent FFL can serve the same function. Becskei and Serrano (2000) show that negative autoregulation in gene circuits can produce stability, whereas Doncic and Skotheim (2013) show that the stability of some cellular states can result from coherent feedforward regulation. Unlike Alon's group, these groups do not explicitly put different motifs together in terms of their common functions. However, their constant and unified use of the common function terms, e.g., 'boosting/speeding up the response time', 'noise suppression/reduction' and 'stability', and their act of attributing the same functions to the distinct motifs, clearly indicate that they have an implicit functional classification system in their mind. In other words, their practice presupposes that there is such a functional classification system. I thank an anonymous referee for highlighting this concern. 
involve two different techniques, designs, or causal stories with respect to removing corks.

Having said this, the next question is whether these factors are also among those that lead the two motifs to be classified as being of the same kind. The key here, according to Polger and Shapiro, is to show that these factors constitute the causally relevant elements with respect to realizing each motif's function. Recall that in the corkscrew example, properties such as color, size, material composition, rigidity, etc., do not count as causally relevant factors with respect to removing corks, while properties such as mechanical principles do count as causally relevant. The analogy between the corkscrew case and the motif case continues. In our motif case, we can also say that properties such as material composition do not concern us, but properties such as how they are causally operating or how components are causally connected do concern us. That is, the causal properties exemplified in each motif are among those that lead each motif to do what it does. They are causally relevant to what the motif can do. Given that the two causal structures end up realizing the same function, it is reasonable to conclude that these causal properties embedded in the two causal structures are among those that lead the two motifs to be classified as being of the same kind. Therefore, to use Shapiro's slogan again, it is the differences that result in the sameness.

It is therefore possible to turn Polger and Shapiro's argument against them. Their original intention in using the corkscrew example was to dismiss the prevalence of MR in 
the biological world, while at the same time admitting its common existence in artifacts. Yet, we have seen from the analogy made above that what they say about corkscrews is equally true of motifs. If the corkscrews achieve the same function through causally relevant but distinct ways, then there is no reason to deny that the motifs also achieve the same function through causally relevant but different ways.

Finally, consider Polger and Shapiro's fourth condition. This condition requires that the relevant differences between the NAR and I1-FFL networks according to the lower taxonomic system must be distinct from the intra-kind variations between them according to the higher taxonomic system. No doubt, the two causal scenarios underlying the two motifs constitute two distinct kinds, as demonstrated above. So, the first half of the fourth condition is satisfied. How about the second half? I think it is also clear that there exist intra-kind variations between the motifs, as in the case of the corkscrews, that differ with respect to their efficiency in removing corks. These intra-kind variations can be easily seen in, as well as predicted from, the two motifs' distinct underlying structures. For instance, the NAR motif's function of boosting the response time is due to its employment of a strong promotor, while the I1-FFLs' execution of the same job owes to the repression factor $\mathrm{F}$, determined by both the maximal and basal activity of the promotor in question (Alon 2007, 61-62). These differences in underlying setups entail that the two motifs are sensitive to different inner molecular milieus (e.g., protein levels), differences in chemical 
bonds, changes of external signals, etc. (even the members within a single type of motif can show variations). In other words, the intra-kind variations between the two motifs are something to be expected. Hence, the second half of the fourth condition is also met.

All in all, our motif case satisfies all the conditions of Polger and Shapiro's recipe, leading us to the conclusion that the NAR and I1-FFL motifs constitute a genuine case of MR.

\section{Are Cases of MR Rare? ${ }^{17}$}

One might be suspicious of the scope of my conclusion, insisting that MR might exist only in a couple of special cases in the biological world. Polger and Shapiro say that "[...] on the best evidence, cases of multiple realization are more like an exception than a rule" $(2018,448)$. This is too hasty if we take seriously the similarity between artifacts and the

17 Note that Robert Batterman $(2000,2014)$ also argues for the generality of MR, though he focuses on cases in thermodynamics and statistical mechanics and makes appeal to universal (i.e., common) behaviors that different systems with heterogeneous underlying mechanisms can exhibit. Also note that, although this essay concentrates on examples drawn from systems biology to support the generality of MR, other authors have discussed a wide variety of examples from, e.g., cognitive science (Wilson and Craver 2006; Walter and Eronen 2014), physics, biology and even social sciences (Ellis 2008; 2011). 
previously overlooked naturally occurring systems, but the opposing case has not been made yet either. Therefore, we had better provide other reasons for thinking that MR is not uncommon in the biological world.

First though, we should be clear about how to proceed. Does 'not-uncommon' refer to the absolute number of instances of MR in the biological world? Obviously not, for if 'not-uncommon' is understood in this way, then it seems MR comes too cheap-if this is the relevant sense, the astronomic number of bacteria such as E. coli instantiating NAR and FFLs has already demonstrated that MR is certainly not uncommon. If 'not-uncommon' does not refer to the number of instances, then how about the number of species that instantiate NAR and FFLs? Evidence of this kind is more promising, for it is able to directly reveal how general the phenomenon is. Happily, systems biologists have shown that many species, such as E. coli, yeasts, and many other higher organisms including humans, instantiate network motifs such as NAR and FFLs (Milo et al. 2002; Mangan and Alon 2003). Moreover, the same network motifs accomplishing important functions can be rediscovered in many different species over long time-scales due to convergent evolution (Conant and Wagner 2003; Babu et al. 2004). ${ }^{18}$

18 It is unclear whether network motifs such as NAR and FFLs, which perform the same function, are results of convergent evolution. Though it does seem clear that the same motifs rediscovered in different species are the outcome of convergent evolution (Conant and Wagner 2003; Babu et al. 2004). Also note that whether a case produced by convergent 
Given this sort of evidence, we may conclude that MR is not rare (in the relevant sense) in the biological world. Also, given that systems biology is a relatively young discipline and that searching for generalizable design principles is its ongoing mission, we may expect to find more species that instantiate NAR and FFLs to be discovered by systems biologists in the near future. Even so, however, this is only one type of evidence-evidence linked with a specific pair of network motifs (i.e., NAR and FFLs) and with only one particular function performed by them. Ideally, we should look to a wider variety of evidence.

First though, a minor point. To be clear, it is not the case that there are only two kinds of motifs that perform the function of boosting the response time of transcription networks. Recall that in Section 3 I mentioned that a simple regulation network can also boost its response time with a very strong promoter or a high degradation rate of the gene product, even though this may generate overexpression of the gene product. Perhaps many other motifs can be found that fulfil the same function.

Second, and more importantly, there are indeed other substantial types of evidence, such as noise suppression, ${ }^{19}$ stability, ${ }^{20}$ and robust patterning in development. ${ }^{21}$ But for evolution counts as a case of MR is an empirical question subject to careful empirical investigation. For discussion of MR related to convergence, see Block and Fodor (1972), Couch (2005, 2018), Polger and Shapiro (2016, Chapter 7).

19 Ghosh et al. (2005) show that noise suppression can be fulfilled by the type-1 coherent 
the sake of brevity, let us focus on robust patterning. During an organism's development, the fertilized single cell must follow different paths in a spatially fine-tuned fashion in order to differentiate into distinct tissues of the organism. Such a sequential process during development is called patterning. How the fertilized single cell can form such a precise spatial pattern depends on a sort of positional information, which is conveyed by morphogen gradients. ${ }^{22}$ The morphogen that is produced and diffused forms a field, and the cells in this field are able to recognize the morphogen via a receptor on the surface of the cells. The fate of a cell depends on which set of genes is expressed in that cell, while which set of genes is expressed in turn hinges on the concentration of morphogen produced

FFL while Singh and Hespanha (2009) show that it can be fulfilled by negative autoregulation.

${ }^{20}$ Becskei and Serrano (2000) show that stability can be realized by negative autoregulation whereas Doncic and Skotheim (2013) show that it can be realized by coherent feedforward regulation.

21 Note that robust patterning in development is a special type of biological robustness, which contains many broader categories of mechanisms that realize the property of robustness, e.g., "system control, alternative (or fail-safe) mechanisms, modularity and decoupling" (Kitano 2004, 827; see also Kitano 2007).

22 "Morphogens are diffusible signaling molecules that pattern cellular fields by setting up differential gene expression in a concentration-dependent manner" (Arias 2003, 321). 
in that cell's environment. At this point, one might reasonably infer that the fate of a cell must be very sensitive to the concentration of morphogen. Nevertheless, biologists have shown that the patterning process is remarkably robust with respect to a whole range of fluctuations and noises including the concentration of morphogen (Eldar et al. 2002, 2004; Moreno-Risueno and Benfey 2011; Morishita and Hironaka 2013).

Technical details aside, what we are interested in here is the question of whether organisms have found different ways (i.e., mechanisms with which) to achieve the function of robust patterning during development. It turns out that robust patterning can be achieved by two different modes of self-enhanced degradation, where self-enhanced degradation is "a feedback mechanism that makes the degradation rate of $M$ [morphogen] increase with the concentration of $M$ " (Alon 2007a, 164). More specifically, systems biologists have discovered two feedback loops (i.e., network motifs) in heterogeneous developmental processes that play the key part in the functioning of robust patterning (for details of robust patterning via self-enhanced degradation, see Eldar et al. $(2002,2003,2004))$, as shown below: 23

${ }^{23}$ Note that, in addition to self-enhanced degradation, there are other mechanisms found to be responsible for robust patterning, e.g., serial transcytosis, pre-steady-state patterning, cell rearrangement, etc. For a survey of these mechanisms, see Wolpert (2011). 


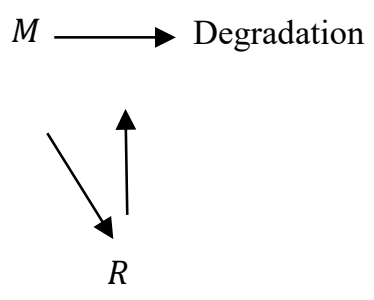

(a)
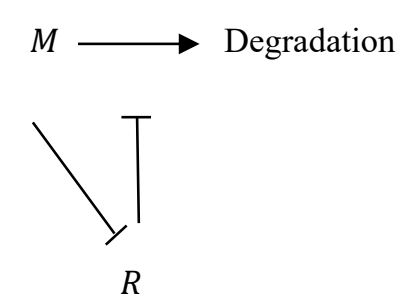

(b)

Figure 5. Two network motifs: (a) self-enhanced degradation via

double-enhancement; (b) self-enhanced degradation via double-inhibiting. This figure comes from Alon (2007a, 166).

In motif (a) morphogen $M$ binds receptor $R$ and enhances its production. Then, $R$ increases the rate at which $M$ is consumed and thus degraded by the cells. In (b) $M$ represses $R$, and then $R$ inhibits a protease that degrades $M$, thus inhibiting $M$ 's degradation. In both cases $M$ 's degradation rate is augmented by itself. A real example of the first motif involves the morphogen 'Hedgehog' and its receptor 'Patched' that are present in the robust patterning process of fruit flies and many other species; a real example of the second involves the morphogen 'Wingless' and its receptor 'Frizzled' that are also present in the robust patterning process of fruit flies (Alon 2007a, 166).

The two motifs constitute two different ways of realizing the same function of robust 
patterning because they involve two different causal mechanisms. The first is a double-enhancement mechanism wherein one element $X$ enhances another element $Y$, which in turn enhances still another element $Z$. The overall effect of this mechanism is reinforced enhancement. The second is a double-inhibiting mechanism where $X$ inhibits $Y$ which in turn inhibits $Z$. The overall effect of this mechanism, however, is also reinforced enhancement. Hence, the effects are the same, though the ways they arrive at them are different. Clearly, this counts as a case of MR if we follow Polger and Shapiro's recipe.

We thus have found an additional and independent type of evidence supporting MR. This new line of evidence involves a different pair of motifs (i.e., double-enhancement vs. double-inhibiting) and a different function (i.e., robust patterning in development). Also, the evidence here involves a range of species including fruit flies, among many others.

Perhaps other lines of evidence can be found too. Nevertheless, the different types of evidence presented in this essay, plus the diversity of species instantiating the motifs and performing their associated functions in each type of evidence, have already demonstrated that MR occurs far more frequently than Polger and Shapiro originally imagined. So, even though we do not know yet if MR is a rule in the biological kingdom, we can at least rest assured that it is certainly not an exception. 


\section{Concluding Remarks}

I have shown that MR is not as uncommon in the biological world as many philosophers believe, in the sense that evidence of different types of MR, ranging over several different species, can be easily found. The NAR and I1-FFL motifs are one type in point, robust patterning in development is another. All in all, systems biology provides us the means with which to discover different design principles that fulfil the same biological functions of interest. Even though the cases I have canvassed here are limited in scope and varied in kind, they at least point to a new direction and serve as a starting point for further investigation.

The more general moral we obtain from these cases is that we should always appreciate the open-ended nature of the biological world. If it is common that biological systems converge into the same underlying structure to perform a function of interest, then there is no a priori reason to exclude the possibility that it is also common for biological systems to diverge into different underlying structures that perform the same function of interest. At the end of the day, of course, it is an empirical question if biological systems have really diverged into different underlying structures that perform the same function, and - in agreement with Polger and Shapiro — any claim for it must be subject to rigorous empirical inspection. But if what this essay has shown is right, we should at least be more 
open to such cases, and question the assertion that MR is a rare phenomenon in the natural world. 


\section{References}

Aizawa, Kenneth. 2009. "Neuroscience and Multiple Realization: A Reply to Bechtel and Mundale.” Synthese 167 (3): 493-510.

Aizawa, Kenneth. 2013. "Multiple Realization by Compensatory Differences.” European Journal for Philosophy of Science 3 (1): 69-86.

Aizawa, Kenneth, and Carl Gillett. 2011. "The Autonomy of Psychology in the Age of Neuroscience.” In Causality in the Sciences, by Phyllis McKay Illari, Federica Russo, and Jon Williamson, 6:202-223. Oxford: Oxford University Press.

Alberghina, Lilia, and Hans V. Westerhoff. 2007. Systems Biology: Definitions and Perspectives. Vol. 13. Springer Science \& Business Media.

Alon, Uri. 2007a. An Introduction to Systems Biology: Design Principles of Biological Circuits. Chapman and Hall/CRC.

Alon, Uri. 2007b. "Network Motifs: Theory and Experimental Approaches." Nature Reviews Genetics 8 (6): 450-461.

Arias, Alfonso Martinez. 2003. "Wnts as Morphogens? The View from the Wing of Drosophila." Nature Reviews Molecular Cell Biology 4 (4): 321-325.

Babu, M. Madan, Nicholas M. Luscombe, L. Aravind, Mark Gerstein, and Sarah A. Teichmann. 2004. "Structure and Evolution of Transcriptional Regulatory Networks." 
Current Opinion in Structural Biology 14 (3): 283-291.

Batterman, Robert. 2000. "Multiple Realizability and Universality." The British Journal for the Philosophy of Science 51 (1): 115-145.

Batterman, Robert. 2014. "Reduction and Multiple Realizability." (Available at the PhilSci-Archive).

Bechtel, William, and Jennifer Mundale. 1999. "Multiple Realizability Revisited: Linking Cognitive and Neural States." Philosophy of Science 66 (2): 175-207.

Becskei, Attila, and Luis Serrano. 2000. "Engineering Stability in Gene Networks by Autoregulation." Nature 405 (6786): 590-93.

Bickle, John. 2003. Philosophy and Neuroscience: A Ruthlessly Reductive Account. Springer.

Bickle, John. 2010. "Has the Last Decade of Challenges to the Multiple Realization Argument Provided Aid and Comfort to Psychoneural Reductionists?" Synthese 177 (2): $247-260$.

Block, Ned J., and Jerry A. Fodor. 1972. "What Psychological States Are Not.” The Philosophical Review 81 (2): 159-181.

Boogerd, Fred, Frank J. Bruggeman, Jan-Hendrik S. Hofmeyr, and Hans V. Westerhoff. 2007. Systems Biology: Philosophical Foundations. Elsevier.

Braillard, Pierre-Alain. 2010. "Systems Biology and the Mechanistic Framework." History 
and Philosophy of the Life Sciences 32 (1): 43-62.

Brigandt, Ingo. 2018. "Explanation of Molecular Processes without Tracking Mechanism Operation.” Philosophy of Science 85 (5): 984-997.

Camas, Francisco M., Jesús Blázquez, and Juan F. Poyatos. 2006. “Autogenous and Nonautogenous Control of Response in a Genetic Network." Proceedings of the National Academy of Sciences 103 (34): 12718-12723.

Chirimuuta, M. 2018. "Marr, Mayr, and MR: What Functionalism Should Now Be About." Philosophical Psychology 31 (3): 403-418.

Clapp, Lenny. 2001. "Disjunctive Properties: Multiple Realizations.” The Journal of Philosophy 98 (3): 111-136.

Conant, Gavin C., and Andreas Wagner. 2003. "Convergent Evolution of Gene Circuits." Nature Genetics 34 (3): 264.

Couch, Mark B. 2004. "Discussion: A Defense of Bechtel and Mundale.” Philosophy of Science 71 (2): 198-204.

Couch, Mark B. 2005. "Functional Properties and Convergence in Biology." Philosophy of Science 72 (5): 1041-1051.

Couch, Mark B. 2009. "Multiple Realization in Comparative Perspective.” Biology \& Philosophy 24 (4): 505-519.

Couch, Mark B. 2018. “Some Concerns with Polger and Shapiro's View.” Philosophical 
Psychology 31 (3): 419-430.

Doncic, Andreas, and Jan M. Skotheim. 2013. "Feedforward Regulation Ensures Stability and Rapid Reversibility of a Cellular State.” Molecular Cell 50 (6): 856-868.

Eldar, Avigdor, Ruslan Dorfman, Daniel Weiss, Hilary Ashe, Ben-Zion Shilo, and Naama Barkai. 2002. "Robustness of the BMP Morphogen Gradient in Drosophila Embryonic Patterning." Nature 419 (6904): 304-308.

Eldar, Avigdor, Dalia Rosin, Ben-Zion Shilo, and Naama Barkai. 2003. "Self-Enhanced Ligand Degradation Underlies Robustness of Morphogen Gradients.” Developmental Cell 5 (4): 635-646.

Eldar, Avigdor, Ben-Zion Shilo, and Naama Barkai. 2004. "Elucidating Mechanisms Underlying Robustness of Morphogen Gradients." Current Opinion in Genetics \& Development 14 (4): 435-439.

Ellis, George FR. 2008. "On the Nature of Causation in Complex Systems.” Transactions of the Royal Society of South Africa 63 (1): 69-84.

Ellis, George FR. 2011. “Top-down Causation and Emergence: Some Comments on Mechanisms." Interface Focus 2 (1): 126-140.

Ghosh, Bhaswar, Rajesh Karmakar, and Indrani Bose. 2005. "Noise Characteristics of Feed Forward Loops.” Physical Biology 2 (1): 36-45.

Gillett, Carl. 2003. “The Metaphysics of Realization, Multiple Realizability, and the 
Special Sciences." The Journal of Philosophy 100 (11): 591-603.

Green, Sara. 2015. "Revisiting Generality in Biology: Systems Biology and the Quest for Design Principles.” Biology \& Philosophy 30 (5): 629-652.

Green, Sara. 2017. "Philosophy of systems and synthetic biology." Stanford Encyclopedia of Philosophy.

https://plato.stanford.edu/entries/systems-synthetic-biology/\#DesiPrinBiol.

Kim, Dongsan, Yung-Keun Kwon, and Kwang-Hyun Cho. 2008. "The Biphasic Behavior of Incoherent Feed-Forward Loops in Biomolecular Regulatory Networks." Bioessays 30 (11-12): 1204-1211.

Kitano, Hiroaki. 2002. “Systems Biology: A Brief Overview.” Science 295 (5560): 16621664.

Kitano, Hiroaki. 2004. “Biological Robustness.” Nature Reviews Genetics 5 (11): 826-837.

Kitano, Hiroaki. 2007. “Towards a Theory of Biological Robustness.” Molecular Systems Biology 3 (1): 137.

Kitcher, Philip. 1984. "1953 and All That. A Tale of Two Sciences.” The Philosophical Review 93 (3): 335-373.

Kollmann, Markus, Linda Løvdok, Kilian Bartholomé, Jens Timmer, and Victor Sourjik. 2005. “Design Principles of a Bacterial Signalling Network.” Nature 438 (7067): 504 507. 
Koskinen, Rami. 2017. "Synthetic Biology and the Search for Alternative Genetic Systems: Taking How-Possibly Models Seriously.” European Journal for Philosophy of Science 7 (3): 493-506.

Latchman, David S. 1997. "Transcription Factors: An Overview." The International Journal of Biochemistry \& Cell Biology 29 (12): 1305-1312.

Levy, Arnon, and William Bechtel. 2013. "Abstraction and the Organization of Mechanisms." Philosophy of Science 80 (2): 241-261.

Mangan, Shmoolik, and Uri Alon. 2003. "Structure and Function of the Feed-Forward Loop Network Motif.” Proceedings of the National Academy of Sciences 100 (21): $11980-11985$.

Mangan, Shmoolik, Alon Zaslaver, and Uri Alon. 2003. "The Coherent Feedforward Loop Serves as a Sign-Sensitive Delay Element in Transcription Networks.” Journal of Molecular Biology 334 (2): 197-204.

Mangan, Shmoolik, Shalev Itzkovitz, Alon Zaslaver, and Uri Alon. 2006. “The Incoherent Feed-Forward Loop Accelerates the Response-Time of the Gal System of Escherichia Coli.” Journal of Molecular Biology 356 (5): 1073-1081.

Milo, Ron, Shai Shen-Orr, Shalev Itzkovitz, Nadav Kashtan, Dmitri Chklovskii, and Uri Alon. 2002. "Network Motifs: Simple Building Blocks of Complex Networks." Science 298 (5594): 824-827. 
Molina, Carlos A., Nicholas S. Foulkes, Enzo Lalli, and Paolo Sassone-Corsi. 1993.

"Inducibility and Negative Autoregulation of CREM: An Alternative Promoter Directs the Expression of ICER, an Early Response Repressor.” Cell 75 (5): 875-886.

Moreno-Risueno, Miguel A., and Philip N. Benfey. 2011. "Time-Based Patterning in Development: The Role of Oscillating Gene Expression.” Transcription 2 (3): 124-129. Morishita, Yoshihiro, and Ken-ichi Hironaka. 2013. "Systems Approach to Developmental Biology-Designs for Robust Patterning." IET Systems Biology 7 (2): 38-49.

Novák, Béla, and John J. Tyson. 2008. "Design Principles of Biochemical Oscillators.” Nature Reviews Molecular Cell Biology 9 (12): 981-991.

O’Malley, Maureen A., and John Dupré. 2005. "Fundamental Issues in Systems Biology.” BioEssays 27 (12): 1270-1276.

Polger, Thomas W. 2009. "Evaluating the Evidence for Multiple Realization.” Synthese 167 (3): 457-472.

Polger, Thomas W., and Lawrence A. Shapiro. 2008. "Understanding the Dimensions of Realization.” The Journal of Philosophy 105 (4): 213-222.

Polger, Thomas W., and Lawrence A. Shapiro. 2016. The Multiple Realization Book. Oxford: Oxford University Press.

Polger, Thomas, and Lawrence Shapiro. 2018. "Responses to Critics.” Philosophical Psychology 31 (3): 446-457. 
Richardson, Robert C. 2008. "Autonomy and Multiple Realization.” Philosophy of Science 75 (5): 526-536.

Rosenfeld, Nitzan, Michael B. Elowitz, and Uri Alon. 2002. "Negative Autoregulation Speeds the Response Times of Transcription Networks." Journal of Molecular Biology 323 (5): 785-793.

Savageau, Michael A. 1976. Biochemical Systems Analysis. A Study of Function and Design in Molecular Biology. Reading, MA: Addison-Wesley.

Shapiro, Lawrence A. 2000. "Multiple Realizations.” The Journal of Philosophy 97 (12): $635-654$.

Shapiro, Lawrence A. 2004. The Mind Incarnate. Cambridge: MIT Press.

Shapiro, Lawrence A. 2008. "How to Test for Multiple Realization." Philosophy of Science 75 (5): 514-525.

Shapiro, Lawrence A., and Thomas W. Polger. 2012. "Identity, Variability, and Multiple Realization in the Special Sciences." In New Perspectives on Type Identity: The Mental and the Physical, ed. Simone Gozzano, and Christopher S. Hill, 264-288. Cambridge: Cambridge University Press.

Shen-Orr, Shai S., Ron Milo, Shmoolik Mangan, and Uri Alon. 2002. "Network Motifs in the Transcriptional Regulation Network of Escherichia Coli." Nature Genetics 31 (1): $64-68$. 
Singh, Abhyudai, and Joao P. Hespanha. 2009. “Optimal Feedback Strength for Noise Suppression in Autoregulatory Gene Networks.” Biophysical Journal 96 (10): 40134023.

Sober, Elliott. 1999. "The Multiple Realizability Argument against Reductionism.” Philosophy of Science 66 (4): 542-564.

Sullivan, Jacqueline Anne. 2008. "Memory Consolidation, Multiple Realizations, and Modest Reductions.” Philosophy of Science 75 (5): 501-513.

Wall, Michael E., Mary J. Dunlop, and William S. Hlavacek. 2005. "Multiple Functions of a Feed-Forward-Loop Gene Circuit.” Journal of Molecular Biology 349 (3): 501-514. Walter, Sven, and Markus Eronen. 2014. "Reduction, Multiple Realizability, and Levels of Reality." In The Bloomsbury Companion to the Philosophy of Science, ed. Steven French and Juha Saatsi, 138-156. Bloomsbury Academic.

Wilson, Robert A., and Carl F. Craver. 2007. "Realization: Metaphysical and Scientific Perspectives.” In Philosophy of Psychology and Cognitive Science, ed. Paul Thagard, 81-104. Amsterdam: North-Holland.

Wolpert, Lewis. 2011. "Positional Information and Patterning Revisited.” Journal of Theoretical Biology 269 (1): 359-365.

Zabet, Nicolae Radu. 2011. "Negative Feedback and Physical Limits of Genes." Journal of Theoretical Biology 284 (1): 82-91. 
Zerilli, John. 2017. "Multiple Realization and the Commensurability of Taxonomies." Synthese, 1-17. 